\title{
The Perceptions of Professional Nurses Regarding the Performance of Cervical Cancer Screening, in Makhuduthamaga Sub-district, Sekhukhune District, Limpopo Province, South Africa
}

\author{
Coshiwe Matildah Makunyane ${ }^{1}$ \\ ${ }^{1}$ Department of Public Health, University of Limpopo, Sovenga, South Africa \\ Correspondence: Coshiwe Matildah Makunyane, Department of Public Health, University of Limpopo, Private \\ Bag X1106, Sovenga, 0727, South Africa. Tel: 27-72-236-4959. E-mail: makunyanec@yahoo.com
}

Received: April 17, 2020 Accepted: September 16, 2020 Online Published: November 17, 2020

doi:10.5539/gjhs.v12n13p152 URL: https://doi.org/10.5539/gjhs.v12n13p152

\begin{abstract}
Cervical cancer is regarded as the most common diagnosed type of cancer and resulting in high cancer related mortality amongst women. Cervical cancer related mortality rate is a serious challenge in Africa as compared to other countries, which needs a high collaborative approach amongst all health professionals. However, the higher incidence and death rate of cervical cancer implies that, high need of cervical cancer screening measures are necessary.

A qualitative, descriptive approach was conducted through focus group discussions to establish perceptions of professional nurses regarding the provision of cervical cancer screening services in clinics. Challenges regarding the provision of cervical cancer services in clinics were raised by professional nurses during interviews. Verbatim data was collected by using interview guides and analyzed using Tesch's 8-step approach in the coding process. Perceptions such as cultural beliefs, lack of resources for conduction of cervical cancer screening and transportation of Pap smear specimens and results, inadequate provision of information and pap smear results to clients were raised by professional nurses as contributing to cervical cancer uptake. Lack of standardized cervical cancer screening training thus leading to professional nurses not being sure of their performance regarding the provision of cervical cancer screening services were also stated by participants as challenges influencing uptake of cervical cancer screening. However, lack of standardized training about cervical cancer screening was found to be affecting the performance on provision of cervical screening services.
\end{abstract}

A need for standardized cervical cancer screening training for all professional nurses is recommended.

Keywords: cervical cancer, cervical cancer screening, cervical cancer screening policy guideline, professional nurses

\section{Introduction}

Cervical cancer remains a second worldwide burden, especially in developing countries like South Africa, where high cervical cancer incidences and mortality rate are common. Invasive cervical cancer (ICC) is said to be the leading cause of cancer related deaths among women in developing countries and second most common cancer among women worldwide (Wright, Aiyedehin, Akinyinka, \& Ilozumba, 2014). Existing estimates show that every year, 14550 women are diagnosed with cervical cancer and 9659 die from the disease in developing countries while 1,5 million cases of cervical cancer are diagnosed globally (Olanlesi-Aliu, Martin, \& Daniels, 2019). However, a decrease of cancer related deaths with an estimation of 7,735 new cases and 4.248 cancer related deaths was observed in 2012 (Jordan et al., 2016). Consideration of the decrease in cancer related deaths implies an improvement which can be brought through strategic interventions which can be collaborated in all staff in the health system. The study adds the body of knowledge about the perceptions of professional nurses regarding the performance of cervical cancer screening in Makhuduthamaga Sub-district.

\section{Background and Literature Review}

Makhuduthamaga Sub-district is situated in Sekhukhune District, which is declared deep rural and the poorest among the five districts in Limpopo Province (Sekhukhune District Draft Development Plan, 2020/2021). Makhuduthamaga Sub-district consists of twenty-one (21) clinics and four (4) mobile clinics where the uptake of 
cervical cancer screening is low, while a high number of women are admitted in hospital at an advanced stage of cervical cancer. According to Sabulei and Maree (2019), South Africa is the second most common cancer in women regarding cervical cancer, and most commonly in black women with $30 \%$ of all cancers. It is expected of every professional nurse to perform their duties as expected by their scope of practice as stated in the Nursing Act 33 of 2005 (Muller \& Bester, 2016) thus fulfilling their functional status (Sabulei \& Maree, 2019). The study was conducted among professional nurses to establish their perceptions regarding performance of cervical cancer in Makhuduthamaga Sub-district, as it contributes and form part of the high cervical cancer statistics in South Africa.

According to Lewis et al. (2007), there is a slow progress of cervical cancer over a period of some years regarding the aetiology and pathophysiology. Sexual exposure of Human Papilloma Virus (HPV), dysplasia and invasive cervical cancer, was stated by Lewis et al. (2007) to be related to repeated injuries to the cervix. A need to improve cervical cancer awareness among the at-risk groups and the menfolk, based on the immense benefit of male involvement in reproductive health matters, has been highly recommended (Wright et al., 2014).

Lack of knowledge about Cervical Cancer Screening was found to be affecting screening programs negatively in South Africa. The lack of awareness about symptoms and consequences of other sexually transmitted infections was supported by several studies conducted among women in rural South Africa. A successful cervical cancer screening for HIV-positive women with high coverage and follow-up of Pap smear positive results was proved.to be achieved through the identification of several barriers (Wake et al., 2009). A critical need to inform women about cervical cancer, Pap smear testing and HPV is recommended to reduce the burden of cervical cancer and save lives of women (Samira et al., 2015).

Consideration and recognition of an important role played by professional nurses in clinics as the front line of the Public Health Sector through conduction of cervical cancer awareness campaigns can be a better strategy to intervene on lack of knowledge.

Low levels of cervical cancer awareness and poor knowledge about cervical cancer including the unavailability and inaccessibility of cervical cancer screening services are said to be contributing to the low uptake of cervical cancer screening in developing countries (Samira et al., 2015) thus leading to high cancer related deaths. Further emphasis is made by Wright et al. (2014), by stating that, cervical cancer morbidity and mortality can be reduced by prevention of cervical cancer which can be achieved through HPV prevention, early detection and treatment. However, Pap smear test is regarded as one of the most reliable and effective cancer screening tests available.

South Africa has therefore introduced a cervical cancer screening policy to ensure that all women between the ages 30 and 70 access the screening services in the public sector. This study, therefore explored the perceptions of professional nurses regarding the provision of cervical cancer screening services in ten clinics of Makhuduthamaga Sub-district.

\section{Method}

\subsection{Design}

The qualitative method was used in the study to obtain information from professional nurses through focus group discussion. Qualitative descriptive design was used to enable the researcher to establish detailed richly described data that was stated by professional nurses regarding their knowledge and perception on provision of cervical cancer screening which is based on their real experiences (Polit \& Beck, 2012; De Vos et al., 2011). The design enables the researcher to obtain all the required information through direct contact with an individual or a group in possession of the knowledge and experiences needed (De Poy \& Gilson, 2008).

\subsection{Study Setting}

The research was conducted in ten randomly selected Makhuduthamaga Sub-district clinics to provide maximum representation. In each of the ten selected clinics of Makhuduthamaga Sub-district at different times, a focus group discussion with professional nurses was conducted. As their contribution impacts on the provision of cervical cancer screening services, feedback from trained nurses were necessary for the study (Krueger \& Casey, 2009).

\subsection{Population and Sampling}

Purposive sampling was used for selection of professional nurses for focus group discussions in February 2015. Professional nurses working in the same (ten) clinics were used as focus groups regardless of gender. In the study, participants ranged between three and six (Total $=46)$ which made it easy for the researcher to manage them during the discussions (Krueger \& Casey, 2009). A total number of forty-six professional nurses from the ten clinics of Makhuduthamaga Sub-district constituted the study population, 


\subsection{Data Collection}

The researcher contacted the Operational Managers' of each clinic who assisted by gathering the professional nurses in a quiet and conducive clinic setting before the focus group discussions. The researcher warmly greeted participants in a semi-circular sitting arrangement and applied the inductive process to facilitate the discussions.

Participants were briefed about the research and signed voluntary participation consent forms. Focus group discussions were conducted in English, which was a widely spoken language by participants, and a tape recorder was also used during the discussions to collect all information. Qualitative data was collected until saturation was reached (Rebar et al., 2011).

Ten focus group discussions were held at various Makhuduthamaga Sub-district clinics. The researcher avoided biases and prejudices which allowed participants to contribute freely and share their views during the discussions for a period of two weeks which lasted for 50 minutes to 1 hour. The moderation styles such as, the process facilitation and the role retraction styles were applied by the researcher to manage the focus group discussion (Breakwell et al., 2009). Participants' responses were recorded and analyzed thematically.

\subsection{Data Analysis}

Data analysis is explained as a systematic organization and synthesis of research data into smaller and more manageable units in order to facilitate understanding (Polit \& Beck, 2012). Recorded data from participants were transcribed and analyzed according to themes and sub-themes to classify responses.

\subsection{Trustworthiness}

Connolly (2016) stated that trustworthiness or rigor of qualitative design is the degree of trust in the data produced, analysis, and methods to improve the truth and integrity of the results. Four qualitative approaches i.e. credibility, confirmability, dependability and transferability were applied to enhance trustworthiness.

\subsubsection{Credibility}

Credibility of results is influenced by the researcher's ability to decide on the focus of the study, selection of the context, the participants and techniques of gathering data (Letsie, 2019). The researcher allowed 50 minutes to an hour to engage fully with participants, where member checking and interviewing techniques such as paraphrasing, reflection and nodding were applied (Letsie, 2019).

\subsubsection{Confirmability}

Confirmability was ensured by transcription, translation, co-coding and on-going review of the study by the researcher through the support of the supervisor (Polit \& Beck, 2012).

\subsubsection{Dependability}

Dependability considers factors of stability of data over time (Letsie, 2019). The same interview guide was used to collect data in all ten clinics where a tape recorder was used during data collection. The researcher served as a chairperson and a scriber to transcribe all information during the interview. The supervisor ensured quality of data collected by support and guidance throughout the study.

\subsubsection{Transferability}

Polit and Beck (2012) describes transferability as the degree to which results can be transferred and implemented in other settings. Data collection process and analysis using the same interview guide was applied by the researcher during focus group discussions in all ten clinics.

\subsection{Ethics Approval}

Permission from the relevant authorities was obtained to conduct the study. Voluntary participation in the study, assurance of safety and withdrawal at any time from the study was explained to participants before commencement of the study.

\section{Findings}

The study results are interpreted according to the demographics, themes and sub-themes of the responses of the participants.

\subsection{Demographics of Focus Group Discussions}

The demographics of the focus group discussions are presented to indicate the number of focus group discussions and professional nurses. Table 1 represents the number of professional nurses in focus group discussions. 
Table 1. Number of professional nurses in focus group discussions

\begin{tabular}{lc}
\hline Focus group & Number of Professional nurses \\
\hline 1 & 5 \\
2 & 6 \\
3 & 5 \\
4 & 5 \\
5 & 3 \\
6 & 5 \\
7 & 5 \\
8 & 5 \\
9 & 3 \\
10 & 4 \\
\hline Total & $\mathbf{4 6}$ \\
\hline
\end{tabular}

\subsection{Themes and Sub-themes}

Main themes and sub-themes were used to code findings and classify participants' responses. Group number and participant number e.g. Group 1 Participant 1 (G1/P1), Group 2 Participant 3 (G2/P3) etc., were used to identify participants. The identified themes are observed in Table 2.

Table 2. Themes and Sub-themes of Professional Nurses perceptions about cervical cancer screening

\begin{tabular}{|c|c|}
\hline Themes & Sub-Themes \\
\hline \multirow{15}{*}{ 1. Cervical cancer screening uptake } & 1.1. Low \\
\hline & 1.1.1. Invasion of privacy \\
\hline & $\begin{array}{l}\text { 1.1.2. Lack of resources (communication system, transport, } \\
\text { equipment, etc) }\end{array}$ \\
\hline & 1.1.3. Unavailability of pap smear results \\
\hline & 1.1.4. Cultural beliefs \\
\hline & 1.1.5. Ten years interval \\
\hline & 1.1.6. Personnel's lack of knowledge \& skill \\
\hline & 1.1.7. Shortage of staff \\
\hline & 1.1.8. Long time on screening \\
\hline & 1.1.9. Pap smear misconception \\
\hline & 1.2. High \\
\hline & 1.2.1. Cervical Cancer screening Campaigns \\
\hline & 1.2.2. High Population \\
\hline & 1.2.3. Clinic accessibility \\
\hline & 1.2.4. HIV positive status \\
\hline \multirow{5}{*}{ 2. Information to women } & 2.1. Informed \\
\hline & 2.1.1. Individual \& group information \\
\hline & 2.1.2. Media \\
\hline & 2.1.3. Promotional material \\
\hline & 2.1.4. Bulletin board \\
\hline
\end{tabular}


2.2. Uninformed

2.2.1. Low literacy level

2.2.2. No cancer screening campaigns

2.2.3. Unavailability of resources

\subsection{Cancer early detection}

3. Cervical cancer screening importance

3.2. Screening vital in Primary health care

3.3. Early detection \& diagnosis of sexually transmitted diseases (STI's)

4.1.1. Easy

4.1.1.1. Cervix easily visualized

4.1.1.2. Adequacy rate good

4.1.1.3. Instrument easy to use

4.1.1.4. Clients prefer female nurses than males

4.1.1.5. Staff trained

4.1.2. Difficult

4.1 Cervical cancer screening Performance

4.1.2.1. Staff not trained

4.1.2.2. Pap smear results not received

4.1.2.3. Competency not certified

4.1.2.4. Replacement of a brush with a spatula

4.1.3. Not sure

4.1.3.1. Skill easy but results adequacy poor

4.1.3.2. Skill not part of nurses training

4.1.3.3. No certification of competence

4.2.1. Less than 5 minutes (x3groups)

4.2 Screening Duration

4.2.2. 5-10 minutes ( $\mathrm{x} 3$ groups)

4.2.3. 10-20 minutes ( $\mathrm{x} 3$ groups)

4.2.4. 20-40miutes (x1group)

4.3.1. 10 years

4.3.2. 5 years

4.3.3. 3 year

4.3 Follow up screening

\subsubsection{Yearly}

4.3.5. 6 Monthly

4.3.6. 6 Months Post delivery

4.3.7. Depends on HIV status

4.3.8. 6 Monthly for HIV Positive clients

5. National Cervical Cancer Screening Policy Guideline implementation.

6. Cervical cancer screening training/in-service

5.1. Policy clearly understood and implemented

5.2. Never seen the policy

6.1. 2 Days workshop

6.2. 1 Day

6.3. In-serviced

6.4. Not in-serviced 
7.1. Biannual cervical cancer screening training conduction

7. Participants' recommendation
7.2. Disposable vaginal speculums

7.3. Standardization of cervical cancer screening skill in professional nurses training

\subsubsection{The Uptake of Cervical Cancer Screening}

Cervical cancer screening practices were reported to be poor in a South African survey. Inspite of good knowledge on cervical screening and readily accessible facilities with available screening services, most women from higher social and educational backgrounds do not undergo cervical screening (Wright et al., 2014). Professional nurses in clinics as the front line of public health care service who provide the promotional and preventive health practices are expected to motivate women for cervical cancer screening as cervical cancer is a burden worldwide. However, the uptake continues to differ according to clinics due to reasons as stated by participants in this study:

G3/P1: "... we are serving a large population and our clinic is easily accessible ...."

G7/P1: "It is high. "Because I have seen them doing some campaigns ...."

G1/P4: "Low. The 10 years interval is long...."

Older women have declined to be screened by young professional nurses due to cultural values and age disparities between nurses and women: G1/P4: "Isn't it in our culture you must respect the old ladies, so immediately you talk about Pap smear exposing their private parts is an insult." G1/P2: "Its an invasion of their privacy by young nurses."

Visiting clinics for Pap smear results was reported to be a challenge which resulted in women not screening for cervical cancer and stated that: G1/P1: "......those who have done Pap smear don't get results, some are discouraged because they don't get the results. We do not have phones to enquire. We just inform the courier to bring them (results) but they don't come."

The uptake of cervical cancer screening was reflected by participants as influenced by lack of knowledge and the skill of conducting cervical cancer screening: G4/ P1: "Its low following the lack of knowledge and skill of personnel."

The cervical cancer screening uptake in clinics of Makhuduthamaga Sub-district was reflected as being affected by the lack of resources and raised a concern to participants: G4/P3:" Shortage of resources."

G5/P4: "The uptake is very low... There is not enough time, as the clinic is always full... but we fail.... we have only two professional nurses ....")

Participants raised cervical cancer screening as painful and treating STIs as a myth raised by women and affecting the uptake of cervical cancer screening negatively: G7/P2: “.... the perception of women to Pap smear because some still think is painful...." G7/P5: ".... I have noted that people .... only request Pap smear when they have STI ..."

\subsubsection{Cervical cancer screening information to women}

The value of health education about cervical cancer screening through its flexibility in accommodating women's preferences, has been regarded by Bansil et al. (2014) as other means of improving the uptake of cervical cancer screening. Participants confirmed information giving as dominant in the Makhuduthamaga Sub-district where other means are used as follows:

(G1/P5: "We give them pamphlets that inform them about cervical cancer screening." G9/P1: "Every morning we are giving health education....." G9/ P5: “...in the consulting rooms we give health education about Pap smears ...."

Participants in the study view the use of media and bulletin boards as a way of educating women about cervical cancer screening as influencing the uptake of cervical cancer screening. The responses of participants were as follows about the media:

G1/P3: “... they just come and ask say they heard from the media and ... ask .... to do them Pap smear.” G7/P4: ".... are using the board which is written all services that the clinic offers...."

Although participants in focus group discussions in different clinics raised their views about information given to women about cervical cancer screening in their clinics, some differed in opinions: 
G10/ P1: “... not all of them are informed .... we give them only when we do campaigns ..., so we do not conduct campaigns in our clinic to be honest ...." G4/P2: "I think it can be some in the sense that the level of literacy, level of education in the community is low ...."

Lack of resources was identified as impacting negatively towards professional nurses in rendering the cervical cancer screening services. Participants' responses, viewed the provision of cervical cancer screening services as a challenge to professional nurses, as reflected below:

G10/P4: “.... even though we give information about cervical cancer screening, they take all speculums from one clinic to the other for the campaign .... community comes to do cervical cancer after the campaigns speculums won't be available... disposable speculums ... when ordered ... are not delivered..."

Policy makers' focus in addressing challenges such as, lack of resources in clinics of Makhuduthamaga Sub-district was found necessary in the study for quality service provision by professional nurses especially where culture was perceived to be an obstacle as:

G1/P4: “... community ... respect their culture and if you tell them about Pap smear, they feel you are undermining their culture." "'.. immediately you talk about Pap smear exposing their private parts it is another thing. " G10/P4: ".... the attitude of older people refusing to be seen by young nurses when naked.".

The results in the study show a need to put more effort into addressing women's lack of knowledge to improve the uptake of cervical cancer screening.

\subsubsection{Importance of Cervical Cancer Screening}

All participants in focus groups discussions, perceived cervical screening as important for various reasons such as: G1/P5: “... primary health care ... deals with primary prevention, so it is important to screen than to treat." G5/P5: “... screen clients at risk of cervical cancer, for early detection of cervical cancer and identify clients with cervical cancer."

Cervical cancer was viewed by participants as dangerous and reflected statements such as:

G9/P1: “Ja, cervical cancer screening is important ... in order to prevent cancers." G6/P4: "It is important ..., we can diagnose/detect cancer .... STIs early but if we do not do pap smears, we can detect it late.”)

In the study, cervical cancer screening was regarded by participants as other means of diagnosing unreported cases of STIs and women well-being as indicated in the response:

G6/P1: “... we can diagnose sexually transmitted diseases that are not reported.".

\subsubsection{Cervical Cancer Screening Performance}

Participants raised different opinions about the skill of conducting cervical cancer screening, following training which is said to be beneficial to professional nurses. However, training on cervical cancer screening skill is crucial as participants were not sure of their performance and results interpretation is a challenge as participants doubted their skill by stating that: G1/P5: "Easy. ... the cervical Os is easy to detect." G7/P4: "Yes... our facility does almost 98\% of endocervical component present ....... it is not a difficult procedure, .... is the easiest for us...." G10/P3: "... we are sure that we have the skill but when the results come, they say the endocervical component is absent, so we tend to doubt our skill." G3/P5: "To others it can be difficult because they are not yet trained...."

Some participants' opinions raised gender issues as affecting cervical cancer screening while others stated that performing the skill is easy. The following were participant's responses: G9/P3: “.... is easy because our clients prefer the female professionals .... unlike the male ones ...." G4/P4: "Not always easy because... results don't come back ...."

The review of professional nurses training, in terms of the curricula was a concern to participants. The inclusion of standardized cervical cancer screening skill in the curricula was reflected by participants as could be a determinant of their competency during conduction of Pap smear tests. However, a concern was raised by participants regarding the unavailability of the brush, which is used for cervical cancer screening and producing quality results unlike the Aylesbury devices which are not approved.

G10/P5: “... this thing was not part of our training during our training, as nurses it was an in-service training, ... one was not certified to be competent because we were only shown on how to do it then you proceed. So, we are not sure if we are competent, we think we are competent because we did not like pass the procedure. " G10/P3: “... the brush and ... I think there is a problem with the spatula that we are using as it is not like, properly designed as there are different spatulas. With the brush the results come back with endocervical component present unlike the 


\section{spatula."}

Performance of cervical cancer screening procedure was viewed differently by participants in the study, where others faced a challenge in obtaining quality cervical smear and said:

G7/P5: "Yes the skill is easy, doing the procedure is easy but .... most of the results come with the endocervical component absent and you might not have done it correctly thus the challenge but doing the whole procedure is not a problem."

The incompetency of professional nurses and the need for standardization of the cervical cancer screening skill was confirmed in the study findings, as participants stated that Pap smear results comes back being inadequate: "the skill is easy but the results are inadequate", "we are not sure if what we are doing is correct as we were never certified if we are doing the correct thing" and "the skill is difficult"

The responses from the participants form part of the answers to the low uptake of cervical cancer screening in Makhuduthamaga Sub-district.The need to standardize the skill for cervical cancer screening in the professional nurses training was further emphasized by participants as follows:

G4P1: “... the procedure and preparation are +/- 10 minutes." G2/P2: "It depends on the size of the woman but all in all I can say 3 minutes." G10/P4: “... I take 1-2 minutes in total is +/- 5 minutes.” G7/P5: "If .... there was proper planning, I think I can take 7 minutes.” G4/P3: “+/- 2 minutes.” G7/P1: "20 minutes for screening.” G4/P4: "+/- 15 minutes including counselling." G7/P4: “... someone may take 7 minutes, someone 15 minutes but honestly the average of a doctor and nurse can take 38 minutes, but the skill is around 25 minutes.”

The importance of follow-up screening according to the cervical cancer screening program and encouragement of women to visit clinics for their cervical cancer screening results was confirmed by participants as follows:

G10/P5: "It is 10 years for normal results and yearly for HIV+ clients." G6/P1: "Their follow-up is 5 years." G5/P2: "It also depends on the HIV status, those with normal results is 3 years and those with abnormal results we follow the recommendations." G8/P2: “... they must come every year." G8/P1: "But for the HIV positive clients we say they must come every six months.” G1/P1: “.... encourage post-natal mothers to do Pap smear after 6 weeks postnatally."

The findings in the study, further emphasized the need to standardize and improve professional nurses' practice, regarding follow-up of women who screened for cervical cancer (Prendergast \& Hunter, 2014). The participants' responses highlighted the need for integrate and collaborate reproductive health service into the HIV programme:

G9/P3: "Conduct training twice per year as we are living in difficult situation for example the condition of HIV/AIDS ... They must conduct training... Age, they say screen age above 30 years, even a child at age 15 years they are sexually active or heredity, reduce age as long as they are sexually active..."

4.2.5 The National Cervical Cancer Screening Policy Guidelines implementation

When screening clients for cervical cancer, the National Cervical Cancer Screening Strategy aims to guide professional nurses. The implementation of the policy was interpreted by the participants differently.

The National Cervical Cancer Screening Policy helps in guiding professional nurses when conducting cervical cancer screening to women. Participants viewed the implementation of the policy differently. (11/46) Some indicated that the policy was inaccessible, never saw it nor were able to talk about it and later requested the policy from the researcher. While some saw the policy as being easy to implement but linked the policy to the lack of resources which made it hard to implement. Responses of the participants were as follows:

G3/P2: "So are you going to make sure that you provide us with enough of the disposable speculums and the policy after the study to distribute?"

When screening clients for cervical cancer, the National Cervical Cancer Screening Strategy is required to guide professional nurses. Participants viewed the implementation of the policy differently. (11/46) Some stated that the policy was inaccessible and were unable to talk about it much and later requested it from the researcher. Participants views regarding the inaccessibility of the cervical cancer screening policy was related to the lack of resources which made its implementation difficult whereas other participants viewed it as simple to implement.

Participants' responses were as follows:

G3/P2:" We have never seen it in the facility." G3/P5: "So are you going to make sure that you provide us with enough of the disposable speculums and the policy after the study to distribute?"

After being asked to express their views on the adoption of the National Cervical Cancer Screening Policy 
Guidelines, participants responded as follows:

G3/P5: "Yes. It is easy even though I am having a suggestion to say how about to have the... the specimens that are ready made to use and discard not the ones that are autoclaved ... I cannot say the name of the clinic, ... if they have the specimen that they use and discard I think it can be accessible."

Although some participants claimed that the policy was inaccessible as expressed in G4 / P4 responses, they were unable to state whether it was simple or difficult to implement the policy. Although some participants viewed the policy as easy to implement, the storage and sharing of vaginal speculums among clinics were their concern. However, disposable vaginal speculums over the sterilised ones were participants' preferences, as stated by G3/P5.

Lack of resources made implementation of the National Cervical Cancer Screening Policy a challenge as reflected in the responses: G5/P1: "To make follow-up is difficult because we do not have telephones to trace clients unless we use home-based carers to trace." G6/P1: "Unless when we do not have resources e.g. lights, in that case we cannot do it." G1/P5: "Sometimes you find that there are example 10 clients who want to do Pap smear and I am 1 professional nurse who should do it, obviously I won't be able to do it, maybe I will give them another return date to come and don't show up. "G1/P4: “... it's difficult because the uptake is low. It also depends on the availability of nurses."

The implementation of the National Cervical Cancer Screening Policy was also an obstacle, primarily due to the 10 -year screening interval as professional nurses were unable to track women due for screening. Participants preferred a shorter screening interval for cervical cancer and expressed their responses as follows:

G5/P5: "The challenge for tracing is for the 10 years clients though not all because others do come and say I have to do Pap smear because is now 10 years, but some do not come." G7/P4: "I think is easy to implement ... but 10 years is too long ... if the private sector do it in 2 years so why can't they do it in 2 years...."

While the implementation of the National Cervical Cancer Screening was seen by some participants as difficult, others saw the policy as easy to implement. The following responses were opinions for participants who found the policy to be easy to enforce but relied on the availability of resources:

G7/P4: "I think is easy to implement... but 10 years is too long.... if the private sector does it in 2 years so why can they not do it in 2 years. But it is easy to implement." G8/P2: "I can say it is easy but sometimes it is a problem because of the shortage of staff... things become easy when you plan."

In the implementation of the National Cervical Cancer Screening Policy Guideline, some participants did not face difficulties and appreciated its use as reflected in the response: G9/P1: "Yes. It is easy to implement... because.... it's easy to use." G2/P3: "Yes. If you have a problem, we in-service each other."

In this study participants showed that the policy is hard to implement and explained their reasons for the difficulties they experienced. Due to the availability of courier services, the issues of not screening women for cervical cancer during public holidays was posed:

G1/P1: "During holidays there is no courier coming so we do not do Pap smear."

The lack of accessibility of women's screening results for cervical cancer in relation to the availability and functionality of telephones in clinics was highlighted as related to the difficulty in implementing the guidelines for the National Cervical Cancer Screening Policy: G1/P5: "No phone for tracing results and clients do not get results.")

The inadequate infrastructure contributing to the lack of privacy and facilities for performing Cervical Cancer Screening was also a concern and was raised as a reason for the low use of screening for cervical Cancer. The following responses were reflected by respondents: G1/P5: "The infrastructure.... where I am working ... there is no curtain for privacy, no fitted lamp, there is no screen so privacy there is compromised and exposing the woman there you find that someone is knocking and without saying come in the person already came in."

The lack of resources seemed to be an underlying obstacle for the study's rendering of the Cervical Cancer Screening services, and while some regarded the policy as easy to enforce, they also referred to the lack of resources as making it difficult to implement the policy.

\subsubsection{Training/In-Service on Cervical Cancer Screening}

Although training requires certain costs and resources to improve the skills of professional nurses, the level of adequacy and cost reduction often depends on skills to perform Cervical Cancer Screening as required by the National Cervical Cancer Screening Policy. The following were responses of participants when asked as if they were trained: 
G5/P3: "I attended a 2 days'workshop." G5/P2: "We were trained in a 1-day workshop." G1/P1: "In serviced." G5/P2: "I was not in serviced."

The Delivery of the Cervical Cancer Screening Service led to all focus group discussions and several suggestions were made about the ten years screening cycle for normal results, lack of funding for disposable speculums and preparation.

\section{Discussion of Results}

Participants shared different opinions on the uptake of cervical cancer screening in group discussions. The responses of participants were considered as important in the study as highlighted by Rubio et al. (2007), that the physician's relationship with patients strengthened the health outcomes associated with improved feelings of satisfaction with treatment.

As little is known about women's awareness of the use of cervical cancer screening, the low uptake of cervical cancer screening is a global concern (Fletcher et al., 2015). Nurse practitioners also play a major role in integrating new guidelines into practice, improving the quality of health care and cancer prevention in women (Schwaiger et al., 2012). In this study, the focus group discussions were conducted by the researcher to encourage professional nurses to their thoughts and perceptions about the low uptake, experiences and practices as a group to direct and influence ach others' views.

Professional nurses are concerned about the low uptake of cervical cancer screening but faced with factors that have a negative effect on uptake, as shown in their responses. Although professional nurses provided different reasons for the low uptake of cervical cancer screening in clinics, the study found it necessary to sensitise professional nurses on the primary prevention of deaths associated with cervical cancer through education, clear communication and financial support, as highlighted by Marek et al., (2011). Some participants, who believed that the use of cervical cancer screening was poor and unreliable, attributed it to the lack of resources and the 10 years interval of cervical screening as very long. More incentives are required to support and encourage staff to screen more women by providing adequate resources to screen women for cervical cancer as a preventive and diagnostic measure for cervical cancer.

According to H. W. Kim and D. H. Kim (2017), the study on: Cervical cancer and sexual lifestyle: a systematic review of health education interventions targeted at women, expects that extra efforts be made to educate all women irrespective of colour or race about HPV and cervical cancer screening, to improve the uptake of cervical cancer screening. Health education was also supported by Bennefield (2015) to be increasing the likelihood of an individual to practice positive health behaviors.

Professional nurses are expected by Pickle et al. (2014) to educate and encourage women about cervical cancer screening. Pickle et al. (2014) described the enhancement of patient awareness of reproductive health topics, the use of culturally relevant patient-centered education materials and cancer screening techniques as ideal for emphasizing cervical cancer to improve women's knowledge on screening.

A research by McLaughlin et al. (2011) found that the published reports showed that the knowledge of Human Papilloma Virus (HPV) and its connection with cervical cancer is increasing. Professional nurses were required to comment on the knowledge of women visiting their clinics if they are accessing cervical cancer screening services.

The findings showed that cultural concerns need to be addressed with the involvement of community structures and stakeholders in order to succeed in the fight against cervical cancer to save women's lives, as culture is also said by Ross et al. (2008) to be playing a role in cervical cancer screening.

The results of the study showed that health education in clinics is performed every day. However, to determine whether there are differences, it is important to study and assess the approaches and knowledge covered in health education. The assessment would be done because health education is supposed to have an impact on people's behavioral change (Prendergast \& Hunter, 2014).

The results also indicate that better health education, emphasizing primary prevention, and the importance of cervical cancer screening could increase the awareness, need, and support of women for cervical cancer screening.

The results show that cultural concerns in relation to cervical cancer need to be discussed in partnership with the community's relevant stakeholders. The results further revealed that, as endorsed by Agurto et al., (2004), mobilization techniques must be introduced with respect to cervical cancer screening. These authors stated that a better way to overcome cultural beliefs as obstacles to cervical cancer screening may be quality communication techniques that consider wider cultural frameworks. Screening for cervical cancer is considered important for all women. Nationally, coordinated cervical cancer prevention programs are considered effective measures to 
minimize cervical cancer-related incidences and mortality rates (Tranberg et al., 2015). The focus group participants were also asked on their point of view, about the significance of cervical cancer screening as a follow-up question as reflected by women.

The results regarding the value of screening for cervical cancer showed that professional nurses are informed, competent and appreciate the need for women to be screened. The knowledge that professional nurses have about cervical cancer screening is basically supposed to be applied through intensified health education and other interventions, such as enhancing the ability to conduct Pap smears and interpret the findings. The rise in the number of cervical cancer screenings and the reduction of cancer-related deaths will be the result of what professional nurses and other health professionals have done with respect to cervical cancer screening. The results revealed that much still needs to be done to ensure that both women are up to date with their cervical cancer screening schedule.

The results about the duration of the screening for cervical cancer showed the difference in the duration of the procedure. Finhaber et al. (2013) emphasized that cervical cancer screening is influenced by cost, patient population, availability of skilled human resources and laboratory capability. Finhaber et al. (2013) also claimed that quality assurance should always be taken into consideration and the best method of screening be determined. The importance of staff training and standardization of the screening protocol for cervical cancer has also been identified in this regard.

Results in this regard showed that most professional nurses are not sure or informed about the follow-up service for cervical cancer screening that they are supposed to provide to clients. According to the National Cervical Cancer Screening Policy Guideline (2013), the results are followed up for 3 to 4 weeks, follow-up screening for normal results is 10 years, while diagnosis is screened for HIV-positive women yearly and three-yearly depending on the current and subsequent screening results (Lince-Deroche et al., 2015).

The results showed that, while participants suggested that it was easy to implement the National Cervical Cancer Screening Policy, some obstacles, such as lack of resources and the ten-year interval, were highlighted by participants as affecting implementation. Sibiya and Graiger (2010) have identified challenges with the implementation of the cervical cancer screening policy in a study on the perceptions of registered nurses of the cervical screening program in primary health care clinics in KwaZulu-Natal Province.

The results indicate that $37 / 46$ ( 80 percent) of the participants were trained or in-serviced about cervical cancer screening. All professional nurses working in clinics are required to be trained for cervical cancer screening. The incompetence of professional nurses about cervical cancer screening conduction in the study, was found to be linked to a lack of training, especially in clinics with large numbers of professional nurses to screen women for cervical cancer. The results revealed lack of training, some participants only in-serviced in the facility, while others attended some workshops away from the clinic with a difference in the training period. The results suggest that all professional nurses require standardized cervical cancer screening.

\section{Conclusion}

Study findings revealed insufficient provision of primary prevention information to women about cervical cancer screening services, resulting in a lack of information and low uptake of cervical cancer screening in the sub-district of Makhuduthamaga. The study also revealed inadequate information / knowledge and obstacles for professional nurses, although the commitment of professional nurses to Cervical Cancer Screening was also observed regardless of lack of training. The findings also showed that the lack of awareness about women's cervical cancer screening is linked to their willingness to screen for cervical cancer. Inadequate Cervical Cancer Screening services was found to be correlated with the unavailability of the cervical cancer screening policy, as reflected by some professional nurses in focus group discussions and the gabs identified in the policy implementation in clinics. The absence of formal cervical cancer screening instruction in the basic training of professional nurses causes difficulties in the provision of screening services.

\section{Limitations of the Study}

- Some professional nurses were on leave at the time of the study, some senior professional nurses or middle managers participated and lack of homogeneity of groups was experienced during the study.

- Some groups were larger than others in different clinics

- Some participants dominated the discussions.

\section{Sources of Funding}

None. 


\section{Acknowledgements}

Special thanks are directed to all professional nurses who have taken part in this study, the Limpopo Department of Health and Makhuduthamaga Sub-district management for the approval of the conduction of the study.

\section{Competing Interests Statement}

The authors declare that there are no competing or potential conflicts of interest.

\section{References}

Agurto, I., Bishop, A., Sanchez, G., Betancourt, Z., \& Robles, S. (2004). Perceived barriers and benefits to cervical cancer screening in Latin America. Preventive medicine, 39(1), 91-98. https://doi.org/10.1016/j.ypmed.2004.03.040

Bansil, P., Wittet, S., Lim, J. L., Winkler, J. L., Paul, P., \& Jeronimo, J. (2014). Acceptability of self-collection sampling for HPV-DNA testing in low-resource settings: a mixed methods approach. BMC public health, 14(1). https://doi.org/10.1186/1471-2458-14-596

Bennefield, Z. C. (2015). Disparities in HPV and cervical cancer screening between highly educated white and minority young women. American Journal of Health Education, 46(2), 90-98. https://doi.org/10.1080/19325037.2014.999963

Breakwell, G. M., Hammond, S., Fife-Schaw, C., \& Smith, J. A. (2009). Research methods in psychology (3rd ed.). London: Sage.

Connolley, L. M. (2016). Trustworthiness in qualitative research. Med Surg Nursing, 25(6), 435-436.

De Vos, A. S., Delport, C. S. L., Fouché, C. B., \& Strydom, H. (2011). Research at grass roots: A primer for the social science and human professions. Van Schaik Publishers.

Department of Health. (2013). National for Cervical Cancer Screening Programme. Pretoria: DoH.

Depoy, E., \& Gilson, S. (2008). Evaluation practice: How-to do-good evaluation research in work setting. London: Routledge Taylor \& Francis Group.

Firnhaber, C., Mayisela, N., Mao, L., Williams, S., Swarts, A., Faesen, M., ... Williamson, A. L. (2013). Validation of cervical cancer screening methods in HIV positive women from Johannesburg South Africa. PloS one, 8(1). https://doi.org/10.1371/journal.pone.0053494

Fletcher, F. E., Buchberg, M., Schover, L. R., Basen-Engquist, K., Kempf, M. C., Arduino, R. C., \& Vidrine, D. J. (2014). Perceptions of barriers and facilitators to cervical cancer screening among low-income, HIV-infected women from an integrated HIV clinic. AIDS care, 26(10). https://doi.org/10.1080/09540121.2014.894617

Jordaan, S., Michelow, P., Richter, K., Simoens, C., \& Bogers, J. (2016). A review of cervical cancer in South Africa: previous, current and future. Health Care Current Reviews, 4(180). https://doi.org/10.4172/2375-4273.1000180

Kim, H. W., \& Kim, D. H. (2017). Awareness of cervical cancer prevention among mothers of adolescent daughters in Korea: Qualitative research. Bio Medical Journal. https://doi.org/10.1136/bmjopen-2014-006915

Krueger, R. A., \& Casey, M. A. (2009). Focus groups: A practical guide for applied research (4th ed.). UK: Sage.

Letsie, T. M. (2019). Transforming South African Public Hospitals Through Intrapreneurship Practice: Views of Unit Nurse Managers Regarding Their Potential Contribution. Global Journal of Health Science, 11(12). https://doi.org/10.5539/gjhs.v11n12p198.

Lewis, S. L., Heitkemper, M. M., Dirksen, S. R., O'Brien, P. G., \& Bucher, L. (2007). Medical surgical nursing: Assessment and management of clinical problems (7th ed.). Missourie, China: Mosby Elsevier.

Lince-Deroche, N. L., Phiri, J., Michelow, P., Smith, J. S., \& Firnhaber, C. (2015). Costs and cost effectiveness of three approaches for cervical cancer screening among HIV-positive women in Johannesburg, South Africa. Plos One, 10(11), eoi41969. https://doi.org/10.1371/journal.pone.0053494

MacLaughlin, K. L., Angstman, K. B., Flynn, P. M., Schmitt, J. R., Weaver, A. L., \& Shuster, L. T. (2011). Predictors of patient comfort and adherence with less frequent cervical cancer screening. Quality in primary care, 19(6), 355-363.

Marek, E., Dergez, T., Kricskovics, A., Kovacs, K., Rebek-Nagy, G., Gocze, K., Kiss, I., Ember, I., \& Gocze, P., (2011). Difficulties in the prevention of cervical cancer: adults' attitudes towards HPV vaccination 3 years 
after introducing the vaccine in Hungary. Vaccine, 29(32), 5122-5129. https://doi.org/10.1016/j.vaccine.2011.05.048

Olanlesi-Aliu, A. D., Martin, P. D., \& Daniels, F. M. (2019). Towards the development of a community-based model for promoting cervical cancer prevention among Yoruba women in Ibadan Nigeria: application of PEN-3 model. Southern African Journal of Gynaecological Oncology, 11(2), 20-24. https://doi.org/10.1080/20742835.2019.1679528

Pickle, S., Altshuler, M., \& Scott, K. C. (2014). Cervical cancer screening outcomes in a refugee population. Journal of Immigrant \& Refugee Studies, 12(1), 1-8. https://doi.org/10.1080/15562948.2013.877698

Polit, D. F., \& Beck, C. T. (2012). Nursing research: Generating and assessing evidence for nursing practice (9th ed.). Philadelphia: Lippincott, Williams \& Wilkins.

Prendergast, M. B., \& Hunter, L. P. (2014). Developing a hand-held Pap test card to improve cervical cancer screening. Nursing for Women's Health, 18(3), 212-219. https://doi.org/10.1111/1751-486X.12122

Rebar, C. R., Gersch, C. J., Macnee, C. L., \& McCabe, S. (2011). Understanding nursing research: Using research in evidence-based practice (3rd ed.). Philadelphia: Wolters Kluwer Health/Lippincott Williams \& Wilkins.

Ross, J. S., Nuñez-Smith, M., Forsyth, B. A., \& Rosenbaum, J. R. (2008). Racial and ethnic differences in personal cervical cancer screening amongst post-graduate physicians: results from a cross-sectional survey. $B M C$ public health, 8(1). https://doi.org/10.1186/1471-2458-8-378

Rubio, R. N., Pearson, H. C., Clark, A. A., \& Breitkopf, C. R. (2007). Satisfaction with care among low-income female outpatients. Psychology, health \& medicine, 12(3), 334-345. https://doi.org/10.1080/13548500600864053.

Sabulei, C., \& Maree, J. E. (2019). An exploration into the quality of life of women treated for cervical cancer. Curationis, 42(1), 1-9. https://doi.org/10.4102/curationis.v42i1.1982

Samira, B., Angelique, M., Abdelkim, \& My, M. (2015). Awareness and knowledge regarding of cervical cancer, Pap smear screening and human papilloma virus infection in Gabonese women. BMC Women's Health Research Article, 2-7.

Schwaiger, C., Aruda, La Coursiere, S., \& Rubin, R. (2012). Current guidelines for cervical cancer screening. Journal of the American Academy of Nurse Practitioners (24), 417-424. https://doi.org/10.1111/j.1745-7599.2012.00704.x

Sekhukhune District Draft Development Plan and Budget 2020/2021. http://www.sekhukhunedistrict.gov.za/sdm-admin/documents/2020-2021,

Sibiya, N., \& Grainger, L. (2010). Registered nurses' perceptions of the cervical screening programme in primary health care clinics in the KwaZulu-Natal Province of South Africa. Africa Journal of Nursing and Midwifery, 12(1), 15-26. https://hdl.handle.net/10520/EJC19343

Tranberg, M., Larsen, M. B., Mikkelsen, E. M., Svanholm, H., \& Andersen, B. (2015). Impact of opportunistic testing in a systematic cervical cancer screening program: a nationwide registry study. BMC Public Health, 15(1). https://doi.org/10.1186/s12889-015-2158-7

Wake, R. M., Rebe, K., \& Burch, V. C. (2009). Patient perception of cervical screening among women living with human immuno-deficiency virus infection attending an antiretroviral therapy clinic in urban South Africa. Journal of Obstetrics and Gynaecology, 29(1), 44-48. https://doi.org/10.1080/01443610802484070

Wright, K. O., Aiyedehin, O., Akinyinka, M. R., \& Ilozumba, O. (2014). Cervical cancer: community perception and preventive practices in an urban neighborhood of Lagos (Nigeria). International Scholarly Research Notices, 2014. https://doi.org/10.1155/2014/950534

\section{Copyrights}

Copyright for this article is retained by the author(s), with first publication rights granted to the journal.

This is an open-access article distributed under the terms and conditions of the Creative Commons Attribution license (http://creativecommons.org/licenses/by/4.0/). 